\title{
Greater Cortical Thickness in Elderly Female Yoga Practitioners-A Cross-Sectional Study
}

\begin{abstract}
Rui F. Afonso ${ }^{1}$, Joana B. Balardin ${ }^{1}$, Sara Lazar ${ }^{2}$, João R. Sato ${ }^{3}$, Nadja Igarashi ${ }^{1}$, Danilo F. Santaella ${ }^{1,4}$, Shirley S. Lacerda ${ }^{1}$, Edson Amaro Jr. ${ }^{1}$ and Elisa H. Kozasa ${ }^{1 \text { * }}$
\end{abstract}

${ }^{1}$ Hospital Israelita Albert Einstein, São Paulo, Brazil, ${ }^{2}$ Massachusetts General Hospital-Harvard Medical School, Boston, MA, United States, ${ }^{3}$ Universidade Federal do ABC, São Bernardo, Brazil, ${ }^{4}$ Centro de Práticas Esportivas da Universidade de São Paulo (CEPEUSP), São Paulo, Brazil

Yoga, a mind-body activity that requires attentional engagement, has been associated with positive changes in brain structure and function, especially in areas related to awareness, attention, executive functions and memory. Normal aging, on the other hand, has also been associated with structural and functional brain changes, but these generally involve decreased cognitive functions. The aim of this cross-sectional study was to compare brain cortical thickness (CT) in elderly yoga practitioners and a group of age-matched healthy non-practitioners. We tested 21 older women who had practiced hatha yoga for at least 8 years and 21 women naive to yoga, meditation or any mind-body interventions who were matched to the first group in age, years of formal education and physical activity level. A T1-weighted MPRAGE sequence was acquired for each participant. Yoga practitioners showed significantly greater CT in a left prefrontal lobe cluster, which included portions of the lateral middle frontal gyrus, anterior superior frontal gyrus and dorsal superior frontal gyrus. We found greater CT in the left prefrontal cortex of healthy elderly women who trained yoga for a minimum of 8 years compared with women in the control group.

Keywords: yoga, cortical thickness, aging, MRI, prefrontal cortex, meditation

\section{INTRODUCTION}

Aging is associated with changes in brain structure and function that may lead to cognitive losses, physical and behavioral changes (Persson et al., 2006; Uranga et al., 2010; Lockhart and DeCarli, 2014). Cognitive impairment in aging includes a decrease in working memory (WM; Draganski et al., 2013; Kirova et al., 2015) and in the volume of cortical areas recruited in attention tasks (Bowman and Dennis, 2015). As longevity steadily increases in the general population worldwide López-Otín et al. (2013), so does the incidence of age-related diseases, leading to higher medical costs (Grootjans-van Kampen et al., 2014). With this in mind, yoga has been considered to be a form of health-management intervention that is non-invasive as well as cost-effective (Aboagye et al., 2015).

Yoga involves the practice of postures, breathing exercises and meditation. While branches of yoga adopted in the West have a strong physical component (postures and breathing exercises), yoga is not limited to the physical body. By definition, yoga is a meditative activity embodied in physical postures in which an attentional component must be present (Taimni, 1961). Therefore, yoga is considered a contemplative practice. 
Its intrinsic and unique contemplative characteristics distinguish it from typical forms of physical exercise, which are limited to the physical body. Ross and Thomas (2010) suggested that yoga has equal or greater effects on various health indicators than regular aerobic exercises. Furthermore, mind-body practices such as yoga, tai chi and meditation have been associated with positive changes in brain structure and function, especially in areas related to awareness, attention, executive functions and memory (Wei et al., 2013; Boccia et al., 2015). Recent studies reported improvement in functional connectivity (verbal, attentional and self-regulatory performance) in relation to verbal memory assessed through the Hopkins Verbal Learning Test-Revised and visuospatial memory measure, the Rey-Osterrieth Complex Test for verbal memory in older adults with mild cognition impairment who underwent yoga interventions (Eyre et al., 2016). Villemure et al. (2015) and Hernández et al. (2016) reported larger gray matter (GM) volumes in practitioners of yoga and Sahaja Yoga Meditation, respectively, compared with controls. To date, however, most studies reporting morphometric differences between mind-body intervention practitioners and non-practitioners have tested small, heterogeneous samples (Boccia et al., 2015; Tang et al., 2015). Moreover, many performed volumetric and region of interest (ROI) analyses and showed mostly mixed results, with different brain regions reported in different studies. There are even fewer studies related to the practice of yoga and morphometric differences in aging. As suggested above, it is important to investigate the effects of yoga in an increasingly older population. Thus, the aim of this study was to compare brain cortical thickness (CT) in elderly female yoga practitioners and healthy non-practitioners.

\section{MATERIALS AND METHODS}

\section{Participants}

Twenty-one female hatha yoga practitioners who practiced at least twice a week for a minimum of 8 years, were recruited from hatha yoga studios in São Paulo, Brazil. Hatha Yoga, one of the most common yoga branches in the West, is based on asana (postures), pranayama (breathing exercise) and dhyana (meditation). We also recruited an additional group of 21 women who were naive to yoga, meditation or any mind-body intervention and were matched to the first group in age, years of formal education and level of physical activity. Subjects were matched for physical activity based on the practices of the Yoga group-those who did not practice any activity other than yoga were matched to sedentary controls and those who practiced yoga plus another physical activity were matched to a control group member who practiced the same or equivalent physical activity. Inclusion criteria were: at least 60 years of age, female, right-handed and having completed at least elementary school. We chose to include only women to add an element of homogeneity to the group. Interestingly, it was also easier to identify female yoga practitioners than male practitioners. Exclusion criteria were: substance abuse; tremor or dystonia of the head; chronic physical or other health problems that prevented them from performing their daily activities independently; any contraindication to MRI; a clinical history of neurological and/or psychiatric diseases. All volunteers provided written informed consent and the study was approved by the Institutional Review Board of Hospital Israelita Albert Einstein (CAAE 22313813.7.0000.0071).

\section{Questionnaires and Tests}

Instrumental Activities of Daily Living-IADL: scores range from 9 (low function) to 27 (high function). Items are evaluated regarding individuals' ability to perform each task (independently, with others' help, or not at all; Lawton and Brody, 1969; Santos and Virtuoso, 2008).

Beck Depression Inventory-BDI: self-report questionnaire with 21 multiple choice questions addressing several depression symptoms. Scores range from 0 to 63 (Beck, 1978; Gorenstein et al., 1999).

Mini Mental State Examination-MMSE: test that evaluates several domains of cognitive function, such as spatial and temporal orientation; calculation; immediate and evoked memory; language-naming; writing; repetition and copying a drawing. Scores range from 0 to 30 (Folstein et al., 1975).

Anthropometric measurements-Weight and height were measured.

\section{Image Acquisition}

A T1-weighted MPRAGE sequence was acquired for each participant using a Siemens 3.0T Magnetom Tim Trio System with a 12-channel head receive coil (matrix $1 \times 1 \times 1 \mathrm{~mm}$ voxel, $\mathrm{TR}=2500 \mathrm{~ms}, \mathrm{TE}=3.45 \mathrm{~ms}, \mathrm{FOV}=265 \mathrm{~mm}$, inversion time $=1100$, flip angle $=7$ degrees). Image quality was visually inspected immediately after each structural acquisition to control for motion effects and other artifacts.

\section{Cortical Thickness (CT) Analysis}

The FreeSurfer analysis suite (v5.3.0 release $^{1}$ ) was used to derive models of cortical surface in each T1-weighted image. These validated and fully automated procedures have been extensively described elsewhere (e.g., Dale et al., 1999; Fischl and Dale, 2000). In brief, a single filled white matter volume was generated for each hemisphere after intensity normalization, skull stripping, and image segmentation using a connected components algorithm. Then, a surface tessellation was generated for each white matter volume by fitting a deformable template. This resulted in a triangular cortical mesh for gray and white matter surfaces consisting of approximately 150,000 vertices (i.e., points) per hemisphere. Measures of CT were computed as the closest distance from the GM and white matter boundary to the GM and cerebrospinal fluid boundary at each vertex on the tesselated surface. Mean CT across the entire brain was also computed for each participant. Thickness data were smoothed using a $15-\mathrm{mm}$ surface-based smoothing kernel.

\footnotetext{
${ }^{1}$ http://surfer.nmr.mgh.harvard.edu/
} 
TABLE 1 | Group characteristics.

\begin{tabular}{|c|c|c|c|}
\hline & Control Group ( $n=21)$ & Yoga Group ( $n=21)$ & $p$ \\
\hline Age (years) & $67.9(1.004)$ & $66.2(0.98)$ & 0.24 \\
\hline Years of Education & $14.6(0.42)$ & $14.1(0.42)$ & 0.35 \\
\hline $\mathrm{BMI}, \mathrm{kg} / \mathrm{m}^{2}$ & $25.3(0.63)$ & $24.5(0.92)$ & 0.53 \\
\hline $\mathrm{BDI}$ & $7.4(1.2)$ & $5.3(0.98)$ & 0.18 \\
\hline MMSE & $28.8(0.28)$ & $28.1(0.38)$ & 0.16 \\
\hline IADL & $26.8(0.14)$ & $26.9(0.04)$ & 0.14 \\
\hline Years of yoga practice & 0.0 & $14.9(1.77)$ & \\
\hline
\end{tabular}

Data expressed as mean (土 standard error); BMI, body mass index; BDI, Beck Depression Inventory; MMSE, Mini Mental State Examination; IADL, Instrumental Activities of Daily Living.

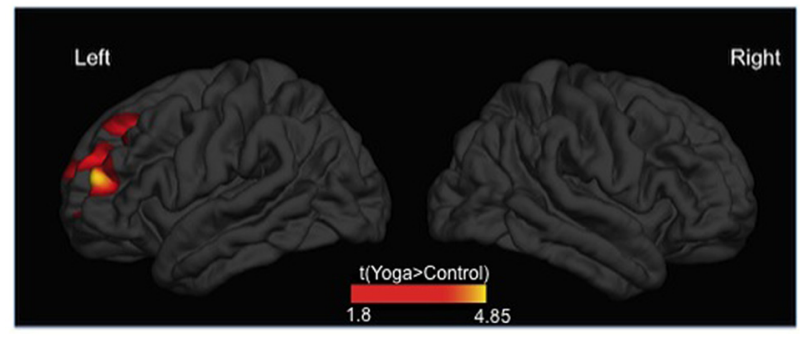

FIGURE 1 | Differences in cortical thickness (CT) between yoginis and controls $(P<0.05$, cluster corrected). Yoga practitioners showed greater CT in a cluster in the left prefrontal cortex (RTF-based, cluster-corrected, $p<0.05$ ).

\section{Statistical Analyses of the Images}

Whole-brain CT analysis was then performed on each vertex using the general linear model (GLM) embedded in the SurfStat toolbox $^{2}$ for Matlab (R2010b; MathWorks). Participants' age was included as a covariate to control for the effect of age on brain structure. CT was compared in yoga practitioners vs. non-practitioners. We also examined different age effects on CT between groups by conducting a group by age interaction. Corrections for multiple comparisons across the whole brain were performed using random-field theory (RFT) cluster-extent based thresholding for nonisotropic images (Worsley et al., 1999). Group differences in mean CT were assessed by an independent samples $t$-test.

\section{Questionnaires and Anthropometric Statistical Analysis}

Data obtained from questionnaires and anthropometric measurements were analyzed using the SPSS 17.0 program (SPSS Inc., Chicago, IL, USA). Variables were compared using Student's $t$ or Mann Whitney tests.

\section{RESULTS}

The yoga group had 14.9 years of hatha yoga practice, on average. There were no significant differences between groups in terms of age, years of education, questionnaire scores or anthropometrical measures, as shown in Table 1.

${ }^{2}$ http://www.math.mcgill.ca/keith/surfstat/

\section{Between-Group Differences in Cortical Thickness}

Relative to controls, yoga practitioners (i.e., female yoga practitioners) showed a significantly greater CT in a left prefrontal lobe cluster (cluster forming threshold $p<0.05$, cluster corrected $p$-value $=0.01574)$, which included anterior and lateral portions of middle and superior frontal gyri (BA8/9; Figure 1, Table 2). No regions exhibited decreased CT in yoginis compared to controls. The age by group interaction was also not significant. There were no significant between-group differences in mean CT for the whole cortex, as assessed for each hemisphere individually $\left(t_{(40)}=-0.690, p=0.494\right)$.

\section{DISCUSSION}

In the present cross-sectional study, we observed greater CT in the left prefrontal cortex (middle and superior frontal gyri) of healthy elderly women who trained yoga for a minimum of 8 years compared with women in the control group. The present results parallel those previously reported in which younger yoga and meditation practitioners had greater GM volumes than non-practitioners compared to non-practitioners in the following brain regions: larger GM volume in the right anterior insula and right inferior temporal gyrus (Hernández et al., 2016), increased GM volume in the frontal lobe (right anterior cingulate cortex and left middle and medial frontal gyrus), left precuneus and fusiform gyrus and right thalamus (Boccia et al., 2015), increased GM volume in the left mid insula, left frontal operculum, left orbitofrontal cortex, right middle temporal gyrus and right primary somatosensory cortex/superior parietal lobule (Villemure et al., 2015). Our sample consisted of healthy elderly Brazilian women with 14.3 years of education, on average (which is high compared to the national average of 4.7 years among people over 60 years of age; IBGE-Instituto Brasileiro de Geografia e Estatística, 2014). The volunteers in this study did not present any severe cognitive impairments, as assessed by the MMSE, nor any problems associated with functionality in daily life activities (as assessed with the IADL). Importantly, no participants displayed any depressive symptoms, as these may be associated with cognitive alterations (McClintock et al., 2010; Dong et al., 2016), or with an early manifestation of dementia (Panza et al., 2010). Therefore, it is unlikely that the differences observed between groups are due to demographic characteristics or depressive symptoms. 
TABLE 2 | Anatomical and statistical information of the cluster in which significant between-group differences in cortical thickness (CT; i.e., yoga practitioners > Controls) were detected.

\begin{tabular}{|c|c|c|c|c|c|}
\hline \multirow[t]{2}{*}{ Region } & \multirow[t]{2}{*}{ Side } & \multicolumn{3}{|c|}{ Talairach/MNI Coordinates } & \multirow[t]{2}{*}{ Peak vertex $t$-value } \\
\hline & & $x$ & $Y$ & $z$ & \\
\hline Middle frontal gyrus & $\mathrm{L}$ & $-35 /-35$ & $47 / 49$ & $10 / 10$ & 4.85 \\
\hline Superior frontal gyrus & $\mathrm{L}$ & $-20 /-20$ & $54 / 57$ & $15 / 17$ & 3.06 \\
\hline
\end{tabular}

* Local maxima within the cluster.

To the best of our knowledge, this is the first imaging study showing greater CT in elderly female yoga practitioners relative to controls. The greater thickness was observed in left prefrontal lobe areas associated with attention and other executive functions (Jeon, 2014). When performing a yoga posture, muscles are engaged for a minimum amount of time in a state of attention (processed in the prefrontal cortex, PFC), similarly to what occurs in meditation (Kane and Engle, 2002; Koechlin et al., 2003). The meditative process (for which attention is essential) is associated with increased oxyhemoglobin concentrations in the PFC due to the increased blood flow to that region (Deepeshwar et al., 2015; Singh et al., 2016). Similarly, alterations in attention and cognition result in different degrees of electrical activity in the PFC (Aftanas and Golocheikine, 2001; Davidson et al., 2003; Lutz et al., 2004; Desai et al., 2015). In our study, we observed alterations in areas associated with executive functions of attentional control rather than motor regions.

Other attentional tasks such as video game playing, cause changes in cognition in older adults (Toril et al., 2014) and brain structure of adolescents, with positive correlation between CT and hour per week of video game in the left dorsolateral prefrontal cortex in left middle frontal gyrus and left frontal eye fields (Kühn et al., 2014). Playing video game may lead to addiction and its deleterious effects, however, yoga is associated with improvement in mental health, therefore different psychobiological mechanisms should be involved in these trainings (Cramer et al., 2013; Pascoe and Bauer, 2015).

Attention-Deficit/Hyperactivity Disorder (ADHD) is related to differences in brain structure and function when compared to controls (Batty et al., 2015; Silk et al., 2016). Yang et al. (2015) and showed thinner superior frontal gyrus in the right hemisphere in children with ADHD compared to healthy controls. Hoekzema et al. (2012) observed reduced laminar CT in a bilateral area from the superior frontal gyrus to the precentral gyrus, inferior and superior parietal cortex. Some of these morphometric abnormalities in ADHD are associated to attention and WM. Furthermore, du Boisgueheneuc et al. (2006) observed that WM was compromised in patients with lesions in the left superior frontal gyrus, one of the regions where we found greater CT in our study. WM allows temporary storage of data and directs attention to relevant information (Baddeley, 1992; Cowan, 2008). It is, therefore, an essential resource of meditative processes, where the object of attention (i.e., breathing) becomes the meaningful content. Moreover, WM capacity decreases with age (Sander et al., 2012). Thus, while aging is associated with a decrease in GM volume in the frontal lobes (Tisserand and Jolles, 2003; Salat et al., 2004; Lockhart and DeCarli, 2014) along with WM deterioration, the practice of yoga appears to have neuroprotective effects, thus having a positive impact on mental health among the elderly. We believe that yoga may preserve cognition, which is supported by our findings as well as previous results in which younger yoga and meditation practitioners showed sharper cognitive functions relative to controls. Eyre et al. (2016) showed that older adults with mild cognitive impairment who practiced 12 weeks of yoga had improvement in functional connectivity (verbal, attentional, and self-regulatory performance) in relation to verbal memory assessed through the Hopkins Verbal Learning Test-Revised and a visuospatial memory measure, the Rey-Osterrieth Complex Test. Considering Taichi chuan a mind-body activity, therefore a mental training, similar to yoga, there were differences in the cortex performing vertex-wise analyses. Wei et al. (2013) reported thicker cortex in Taichi chuan practitioners compared to control group: medial occipitotemporal sulcus, superior temporal gyrus and lingual sulcus in left hemisphere and the circular sulcus of the insula, precentral gyrus and middle frontal sulcus in right hemisphere. Furthermore, Gard et al. (2014) showed that meditation may reduce cognitive decline associated with normal aging, and Sharma et al. (2014) observed greater enhancement in cognitive domains such as memory retention and attention in yoga practitioners relative to controls. Gothe et al. (2014) found that yoga practitioners had greater WM capacity compared with a control group that did stretching and strengthening exercises. In that study, the different results may be due to the attention/awareness component found in yoga but not in stretching and strengthening. Chandra et al. (2016) also reported greater WM capacity in Sudarshan Kriya yoga practitioners. Even though the studies mentioned above detected cognitive alterations, they did not perform imaging tests.

Our study has some limitations. Ideally, individuals across groups should have performed the same physical activities (other than yoga). Matching participants on this variable proved to be very difficult. Thus, we matched as best we could based on relative physical effort. Furthermore, as the number of participants in each group was relatively small, it did not allow us to make comparisons between individuals from different ethnic groups. Also, our volunteers performed only one MRI scan. Future studies should involve longitudinal randomized controlled trials and correlations with other peripheral measures.

In conclusion, healthy elderly women who practiced hatha yoga for at least 8 years had greater prefrontal CT than a group of matched controls. This CT may be associated with cognitive preservation. 


\section{AUTHOR CONTRIBUTIONS}

RFA: acquisition of data; design; interpretation; revising and final approval of the article; agreement to be accountable for all aspects of the work. JBB and JRS: analysis; revising and final approval of the article; agreement to be accountable for all aspects of the work. SL and SSL: interpretation; revising and final approval of the article; agreement to be accountable for all aspects of the work. NI: acquisition of data; final approval of the article; agreement to be accountable for all aspects of the work. DFS: interpretation; final approval of the article; agreement to be accountable for all aspects of the work. EA: revising and final approval of the article; agreement to be accountable for all aspects of the work. EHK: conception and design of the work; analysis and interpretation of data; revising and final approval

\section{REFERENCES}

Aboagye, E., Karlsson, M. L., Hagberg, J., and Jensen, I. (2015). Cost-effectiveness of early interventions for non-specific low back pain: a randomized controlled study investigating medical yoga, exercise therapy and self-care advice. J. Rehabil. Med. 47, 167-173. doi: 10.2340/16501977-1910

Aftanas, L. I., and Golocheikine, S. A. (2001). Human anterior and frontal midline theta and lower alpha reflect emotionally positive state and internalized attention: high-resolution EEG investigation of meditation. Neurosci. Lett. 310, 57-60. doi: 10.1016/s0304-3940(01) 02094-8

Baddeley, A. (1992). Working memory. Science 255, 556-559. doi: 10.1126/science. 1736359

Batty, M. J., Palaniyappan, L., Scerif, G., Groom, M. J., Liddle, E. B., Liddle, P. F., et al. (2015). Morphological abnormalities in prefrontal surface area and thalamic volume in attention deficit/hyperactivity disorder. Psychiatry Res. 233, 225-232. doi: 10.1016/j.pscychresns.2015.07.004

Beck, A. T. (1978). Depression Inventory. Philadelphia, PA: Center for Cognitive Therapy.

Boccia, M., Piccardi, L., and Guariglia, P. (2015). The meditative mind: a comprehensive meta-analysis of MRI studies. Biomed. Res. Int. 2015:419808. doi: $10.1155 / 2015 / 419808$

Bowman, C. R., and Dennis, N. A. (2015). Age differences in the neural correlates of novelty processing: the effects of item-relatedness. Brain Res. 1612, 2-15. doi: 10.1016/i.brainres.2014.08.006

Chandra, S., Sharma, G., Mittal, A. P., and Jha, D. (2016). Effect of Sudarshan Kriya (meditation) on gamma, alpha, and theta rhythm during working memory task. Int. J. Yoga 9, 72-76. doi: 10.4103/0973-6131.171715

Cowan, N. (2008). What are the differences between long-term, short-term, and working memory? Prog. Brain Res. 169, 323-338. doi: 10.1016/S00796123(07)00020-9

Cramer, H., Lauche, R., Langhorst, J., and Dobos, G. (2013). Yoga for depression: a systematic review and meta-analysis. Depress. Anxiety 30, 1068-1083. doi: $10.1002 /$ da. 22166

Dale, A. M., Fischl, B., and Sereno, M. I. (1999). Cortical surface-based analysis. I. Segmentation and surface reconstruction. Neuroimage 9, 179-194. doi: 10.1006/nimg.1998.0395

Davidson, R. J., Kabat-Zinn, J., Schumacher, J., Rosenkranz, M., Muller, D., Santorelli, S. F., et al. (2003). Alterations in brain and immune function produced by mindfulness meditation. Psychosom. Med. 65, 564-570. doi: 10.1097/01.psy.0000077505.67574.e3

Deepeshwar, S., Vinchurkar, S. A., Visweswaraiah, N. K., and Nagendra, H. R. (2015). Hemodynamic responses on prefrontal cortex related to meditation and attentional task. Front. Syst. Neurosci. 8:252. doi: 10.3389/fnsys.2014. 00252

Desai, R., Tailor, A., and Bhatt, T. (2015). Effects of yoga on brain waves and structural activation: a review. Complement. Ther. Clin. Pract. 21, 112-118. doi: 10.1016/j.ctcp.2015.02.002 of the article; agreement to be accountable for all aspects of the work.

\section{FUNDING}

This work was supported by Fundação de Amparo à Pesquisa do Estado de São Paulo (FAPESP; 2015/18262-7), Instituto Israelita de Ensino e Pesquisa Albert Einstein and Instituto de Ensino e Pesquisa em Yoga (IEPY).

\section{ACKNOWLEDGMENTS}

We would like to thank Telma Busch and Michel Naslavsky for their technical support.

Dong, H.-S., Han, C., Jeon, S. W., Yoon, S., Jeong, H.-G., Huh, Y. J., et al. (2016). Characteristics of neurocognitive functions in mild cognitive impairment with depression. Int. Psychogeriatr. 28, 1181-1190. doi: 10.1017/S1041610216000314

Draganski, B., Lutti, A., and Kherif, F. (2013). Impact of brain aging and neurodegeneration on cognition: evidence from MRI. Curr. Opin. Neurol. 26, 640-645. doi: 10.1097/WCO.0000000000000029

du Boisgueheneuc, F., Levy, R., Volle, E., Seassau, M., Duffau, H., Kinkingnehun, S., et al. (2006). Functions of the left superior frontal gyrus in humans: a lesion study. Brain 129, 3315-3328. doi: 10.1093/brain/awl244

Eyre, H. A., Acevedo, B., Yang, H., Siddarth, P., Van Dyk, K., Ercoli, L., et al. (2016). Changes in neural connectivity and memory following a yoga intervention for older adults: a pilot study. J. Alzheimers Dis. 52, 673-684. doi: 10.3233/JAD-150653

Fischl, B., and Dale, A. M. (2000). Measuring the thickness of the human cerebral cortex from magnetic resonance images. Proc. Natl. Acad. Sci. U S A 97, 11050-11055. doi: 10.1073/pnas.200033797

Folstein, M. F., Folstein, S. E., and McHugh, P. R. (1975). "Mini-Mental State:" a practical method for grading the cognitive state of patients for the clinician. J. Psychiatr. Res. 12, 189-198.

Gard, T., Hölzel, B. K., and Lazar, S. W. (2014). The potential effects of meditation on age-related cognitive decline: a systematic review. Ann. N Y Acad. Sci. 1307, 89-103. doi: $10.1111 /$ nyas. 12348

Gorenstein, C., Andrade, L., Vieira Filho, A. H., Tung, T. C., and Artes, R. (1999). Psychometric properties of the portuguese version of the beck depression inventory on brazilian college students. J. Clin. Psychol. 55, 553-562. doi: 10.1002/(SICI)1097-4679(199905)55:5<553::AID-JCLP3>3.3.CO;2-4

Gothe, N. P., Kramer, A. F., and McAuley, E. (2014). The effects of an 8-week Hatha yoga intervention on executive function in older adults. J. Gerontol. A Biol. Sci. Med. Sci. 69, 1109-1116. doi: 10.1093/gerona/glu095

Grootjans-van Kampen, I., Engelfriet, P. M., and van Baal, P. H. (2014). Disease prevention: saving lives or reducing health care costs? PLoS One 9:e104469. doi: 10.1371/journal.pone.0104469

Hernández, S. E., Suero, J., Barros, A., González-Mora, J. L., and Rubia, K. (2016). Increased grey matter associated with long-term sahaja yoga meditation: a voxel-based morphometry study. PLoS One 11:e0150757. doi: 10.1371/journal. pone. 0150757

Hoekzema, E., Carmona, S., Ramos-Quiroga, J. A., Richarte Fernández, V., Picado, M., Bosch, R., et al. (2012). Laminar thickness alterations in the fronto-parietal cortical mantle of patients with attentiondeficit/hyperactivity disorder. PLoS One 7:e48286. doi: 10.1371/journal.pone. 0048286

IBGE-Instituto Brasileiro de Geografia e Estatística. (2014). Síntese de Indicadores Sociais: uma análise das condições de vida da população brasileira. Available online at: http://biblioteca.ibge.gov.br/ visualizacao/livros/liv91983.pdf

Jeon, H.-A. (2014). Hierarchical processing in the prefrontal cortex in a variety of cognitive domains. Front. Syst. Neurosci. 8:223. doi: 10.3389/fnsys.2014.00223 
Kane, M. J., and Engle, R. W. (2002). The role of prefrontal cortex in working-memory capacity, executive attention, and general fluid intelligence: an individual-differences perspective. Psychon. Bull. Rev. 9, 637-671. doi: 10.3758/bf03196323

Kirova, A. M., Bays, R. B., and Lagalwar, S. (2015). Working memory and executive function decline across normal aging, mild cognitive impairment, and Alzheimer's disease. Biomed. Res. Int. 2015:748212. doi: 10.1155/2015/748212

Koechlin, E., Ody, C., and Kouneiher, F. (2003). The architecture of cognitive control in the human prefrontal cortex. Science 302, 1181-1185. doi: $10.1126 /$ science. 1088545

Kühn, S., Lorenz, R., Banaschewski, T., Barker, G. J., Büchel, C., Conrod, P. J., et al. (2014). Positive association of video game playing with left frontal cortical thickness in adolescents. PLoS One 9:e91506. doi: 10.1371/journal. pone.0091506

Lawton, M. P., and Brody, E. M. (1969). Assessment of older people: self-maintaining and instrumental activities of daily living. Gerontologist 9, 179-186. doi: 10.1093/geront/9.3_part_1.179

Lockhart, S. N., and DeCarli, C. (2014). Structural imaging measures of brain aging. Neuropsychol. Rev. 24, 271-289. doi: 10.1007/s11065-014-9268-3

López-Otín, C., Blasco, M. A., Partridge, L., Serrano, M., and Kroemer, G. (2013). The hallmarks of aging. Cell 153, 1194-1217. doi: 10.1016/j.cell.2013.05.039

Lutz, A., Greischar, L. L., Rawlings, N. B., Ricard, M., and Davidson, R. J. (2004). Long-term meditators self-induce high-amplitude gamma synchrony during mental practice. Proc. Natl. Acad. Sci. U S A 101, 16369-16373. doi: 10.1073/pnas. 0407401101

McClintock, S. M., Husain, M. M., Greer, T. L., and Cullum, C. M. (2010). Association between depression severity and neurocognitive function in major depressive disorder: a review andsynthesis. Neuropsychology 24, 9-34. doi: 10.1037/a0017336

Panza, F., Frisardi, V., Capurso, C., D'Introno, A., Colacicco, A. M., Imbimbo, B. P., et al. (2010). Late-life depression, mild cognitive impairment, and dementia: possible continuum? Am. J. Geriatr. Psychiatry 18, 98-116. doi: 10.1097/JGP.0b013e3181b0fa13

Pascoe, M. C., and Bauer, I. E. (2015). A systematic review of randomised control trials on the effects of yoga on stress measures and mood. J. Psychiatr. Res. 68, 270-282. doi: 10.1016/j.jpsychires.2015.07.013

Persson, J., Nyberg, L., Lind, J., Larsson, A., Nilsson, L. G., Ingvar, M., et al. (2006). Structure-function correlates of cognitive decline in aging. Cereb. Cortex 16, 907-915. doi: 10.1093/cercor/bhj036

Ross, A., and Thomas, S. (2010). The health benefits of yoga and exercise: a review of comparison studies. J. Altern. Complement. Med. 16, 3-12. doi: 10.1089/acm. 2009.0044

Salat, D. H., Buckner, R. L., Snyder, A. Z., Greve, D. N., Desikan, R. S., Busa, E., et al. (2004). Thinning of the cerebral cortex in aging. Cereb. Cortex 14, 721-730. doi: 10.1093/cercor/bhh032

Sander, M. C., Lindenberger, U., and Werkle-Bergner, M. (2012). Lifespan age differences in working memory: a two-component framework. Neurosci. Biobehav. Rev. 36, 2007-2033. doi: 10.1016/j.neubiorev.2012.06.004

Santos, R. L., and Virtuoso, J. S. Jr. (2008). Confiabilidade da versão brasileira da escala de atividades instrumentais da vida diária. RBPS 21, 290-296. doi: 10.5020/18061230.2008.p290
Sharma, V. K., Rajajeyakumar, M., Velkumary, S., Subramanian, S. K., Bhavanani, A. B., Madanmohan, et al. (2014). Effect of fast and slow pranayama practice on cognitive functions in healthy volunteers. J. Clin. Diagn. Res. 8, 10-13. doi: 10.7860/JCDR/2014/7256.3668

Silk, T. J., Beare, R., Malpas, C., Adamson, C., Vilgis, V., Vance, A., et al. (2016). Cortical morphometry in attention deficit/hyperactivity disorder: contribution of thickness and surface area to volume. Cortex 82, 1-10. doi: 10.1016/j.cortex. 2016.05.012

Singh, K., Bhargav, H., and Srinivasan, T. M. (2016). Effect of uninostril yoga breathing on brain hemodynamics: a functional near-infrared spectroscopy study. Int. J. Yoga 9, 12-19. doi: 10.4103/0973-6131.171711

Taimni, I. K. (1961). The Science of Yoga. Wheaton, IL: The theosophical publishing house.

Tang, Y.-Y., Hölzel, B. K., and Posner, M. I. (2015). The neuroscience of mindfulness meditation. Nat. Rev. Neurosci. 16, 213-225. doi: $10.1038 / \mathrm{nrn} 3916$

Tisserand, D. J., and Jolles, J. (2003). On the involvement of prefrontal networks in cognitive ageing. Cortex. 39, 1107-1128. doi: 10.1016/s0010-9452(08)7 0880-3

Toril, P., Reales, J. M., and Ballesteros, S. (2014). Video game training enhances cognition of older adults: a meta-analytic study. Psychol. Aging 29, 706-716. doi: $10.1037 / \mathrm{a} 0037507$

Uranga, R. M., Bruce-Keller, A. J., Morrison, C. D., Fernandez-Kim, S. O., Ebenezer, P. J., Zhang, L., et al. (2010). Intersection between metabolic dysfunction, high fat diet consumption, and brain aging. J. Neurochem. 114, 344-361. doi: 10.1111/j.1471-4159.2010.06803.x

Villemure, C., čeko, M., Cotton, V. A., and Bushnell, M. C. (2015). Neuroprotective effects of yoga practice: age-, experience- and frequencydependent plasticity. Front. Hum. Neurosci. 9:281. doi: 10.3389/fnhum.2015. 00281

Wei, G.-X., Xu, T., Fan, F.-M., Dong, H.-M., Jiang, L.-L., Li, H.-J., et al. (2013). Can Taichi reshape the brain? A brain morphometry study. PLoS One 8:e61038. doi: 10.1371/journal.pone.0061038

Worsley, K. J., Andermann, M., Koulis, T., MacDonald, D., and Evans, A. C. (1999). Detecting changes in nonisotropic images. Hum. Brain Mapp. 8 , 98-101.

Yang, X. R., Carrey, N., Bernier, D., and MacMaster, F. P. (2015). Cortical thickness in young treatment-naive children with ADHD. J. Atten. Disord. 19, 925-930. doi: 10.1177/1087054712455501

Conflict of Interest Statement: The authors declare that the research was conducted in the absence of any commercial or financial relationships that could be construed as a potential conflict of interest.

Copyright (C) 2017 Afonso, Balardin, Lazar, Sato, Igarashi, Santaella, Lacerda, Amaro and Kozasa. This is an open-access article distributed under the terms of the Creative Commons Attribution License (CC BY). The use, distribution or reproduction in other forums is permitted, provided the original author(s) or licensor are credited and that the original publication in this journal is cited, in accordance with accepted academic practice. No use, distribution or reproduction is permitted which does not comply with these terms. 